\title{
Intraluminal duodenal ("windsock") diverticulum: a rare cause of biliary obstruction and acute pancreatitis in the adult $\square$
}

(이요 $\odot$

\author{
Authors \\ Institutions \\ 1 Department of Interventional Gastroenterology, \\ Acibadem City Clinic Tokuda Hospital, Sofia, Bulgaria \\ 2 Department of Surgery, University Hospital "Saint \\ Marina”, Pleven, Bulgaria \\ submitted 24.5.2018 \\ accepted after revision 8.10 .2018 \\ Bibliography \\ DOI https://doi.org/10.1055/a-0808-3834 | \\ Endoscopy International Open 2019; 07: E87-E89 \\ (c) Georg Thieme Verlag KG Stuttgart · New York \\ ISSN 2364-3722 \\ Corresponding author \\ Petko Karagyozov, MD, Department of Interventional \\ Gastroenterology, Clinic of Gastroenterology, Acibadem \\ City Clinic Tokuda Hospital, 51B N.Vaptzarov Blvd., 1407 \\ Sofia, Bulgaria
}

Petko Karagyozov¹, Ivan Tishkov ${ }^{1}$, Zhenya Georgieva', Irina Boeva', Dimitar Tzankov²

Fax: +359-2-8155205
petko.karagyozov@gmail.com

\section{ABSTRACT}

An intraluminal duodenal diverticulum (IDD) is a rare congenital anomaly, which is a result of incomplete recanalization of the foregut lumen during embryonic development. Most patients are asymptomatic. Symptoms usually occur after the third decade of life and mainly include epigastric pain, nausea, vomiting, or bloating. Less commonly, IDD may complicate with bleeding, duodenal obstruction, or acute pancreatitis. We present a case of IDD, manifested for a first time in adult with acute biliary obstruction and mild pancreatitis after laparoscopic cholecystectomy for acute calculous cholecystitis, successfully managed with endoscopic retrograde cholangiopancreatography (ERCP).

\section{Introduction}

An intraluminal duodenal diverticulum ("windsock" diverticulum) is a rare congenital abnormality related to an incomplete recanalization of the foregut during embryonic development and resulting in a duodenal diaphragm or web. Years of peristalsis lead to the development of a pulsion-like diverticulum centrally from the duodenal web $[1,3]$. Symptoms usually appear after the age of 30 and are nonspecific including nausea, early satiety, or abdominal pain. Rare presentations are acute pancreatitis, acute upper gastrointestinal bleeding or upper gastrointestinal obstruction [2,3]. It was first described by Silock in 1885 [1].

\section{Case report}

A 72-year-old woman was admitted to our unit with worsening abdominal pain, dark urine, and vomiting 6 days after laparoscopic cholecystectomy for acute calculous cholecystitis. Laboratory work-up revealed elevated liver enzymes, C-reactive protein (CRP), and lipase levels. Abdominal ultrasound showed a dilated common bile duct (CBD), slight pancreatic edema, and a biloma in the gallbladder bed. A computed tomography (CT) scan confirmed the findings and a cystic tumor mass was suspected in the duodenum at the level of the ampulla of Vater ( Fig. 1, $>$ Fig.2). Magnetic resonance cholangiopancreatography (MRCP) showed a small $(5 \mathrm{~mm})$ stone in the CBD ( Fig.3). The patient was scheduled for endoscopic retrograde cholangiopancreatography (ERCP). A double lumen was seen in the second portion of the duodenum, corresponding to a large saccular structure surrounded on both sides by normal mucosa ( $\triangleright$ Fig. 4, $\vee$ Video 1 ). The papillary orifice was difficult to find at the level of the septum ( $\triangleright$ Fig.5). Cholangiography showed a dilated CBD and intrahepatic ducts, stenosis at the level of the papilla, and no leakage from the cystic duct stump. Biliary sphincterotomy followed by papillary large balloon dilation to $12 \mathrm{~mm}$ was performed. Balloon sweeps did not show any stones. The procedure was followed by duodenal exploration using a standard gastroscope and showing the diverticulum 


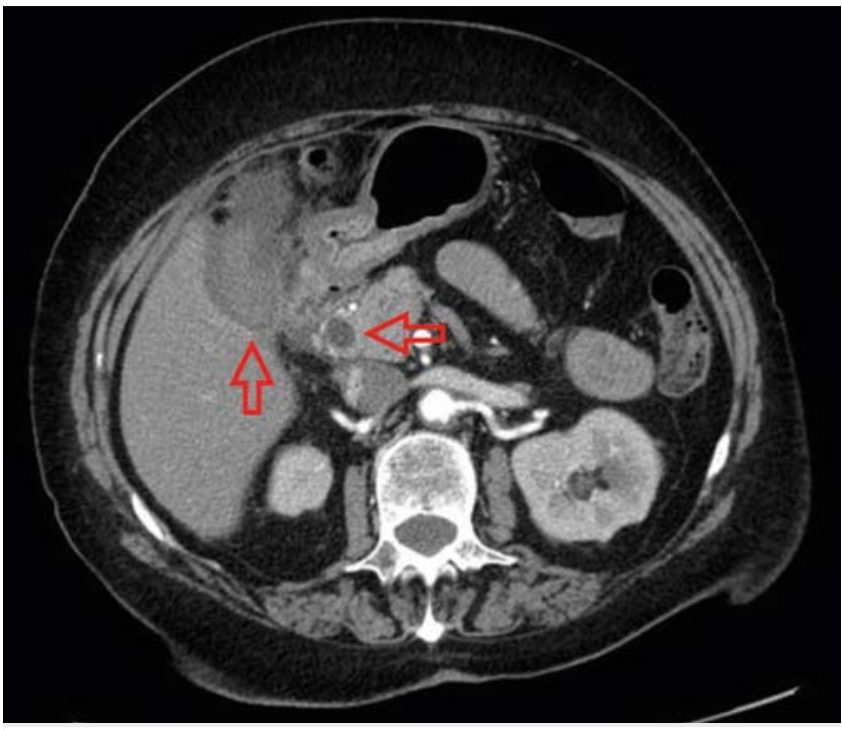

- Fig. 1 Computed tomography (CT) scan showing dilated common bile duct (CBD) and biloma in the gallbladder bed.

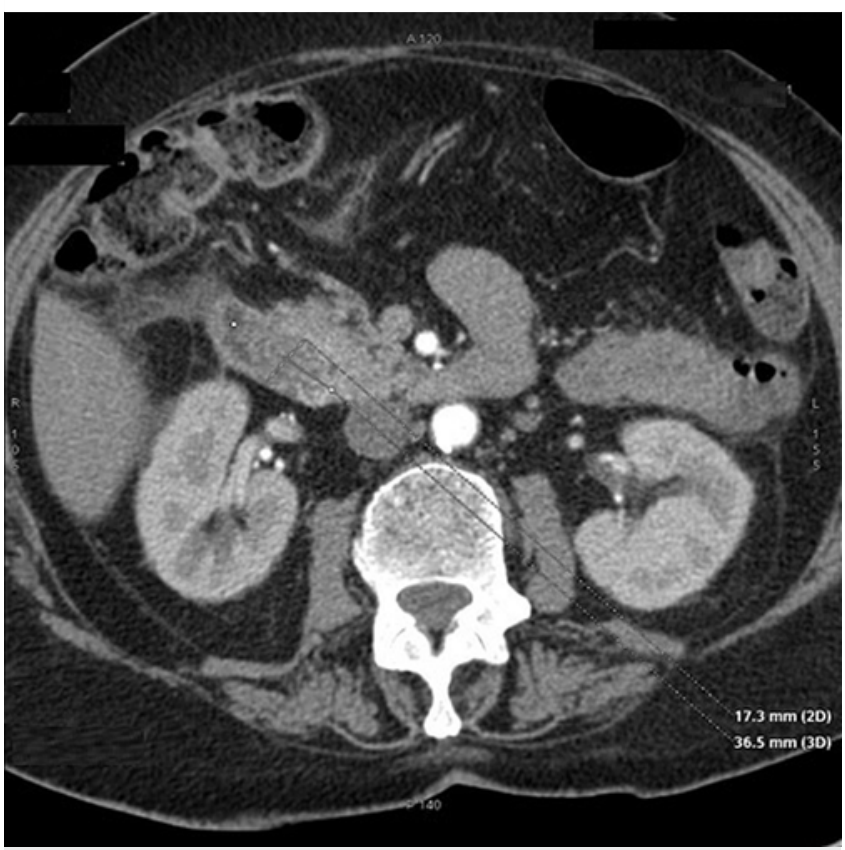

- Fig. 2 Computed tomography (CT) scan showing a suspected cystic tumor mass at the level of the ampulla of Vater.

( $\vee$ Video 1). The condition of the patient rapidly improved. Two weeks later, the biloma had completely disappeared.

\section{Discussion}

An intraduodenal diverticulum ("windsock" diverticulum) is a rare condition, which can be difficult to diagnose. The typical endoscopic finding is a double lumen in the second portion of the duodenum just below the level of the papilla of Vater. The endoscopic appearance may be challenging - the diverticulum may be collapsed and missed or a long sack may be mistaken

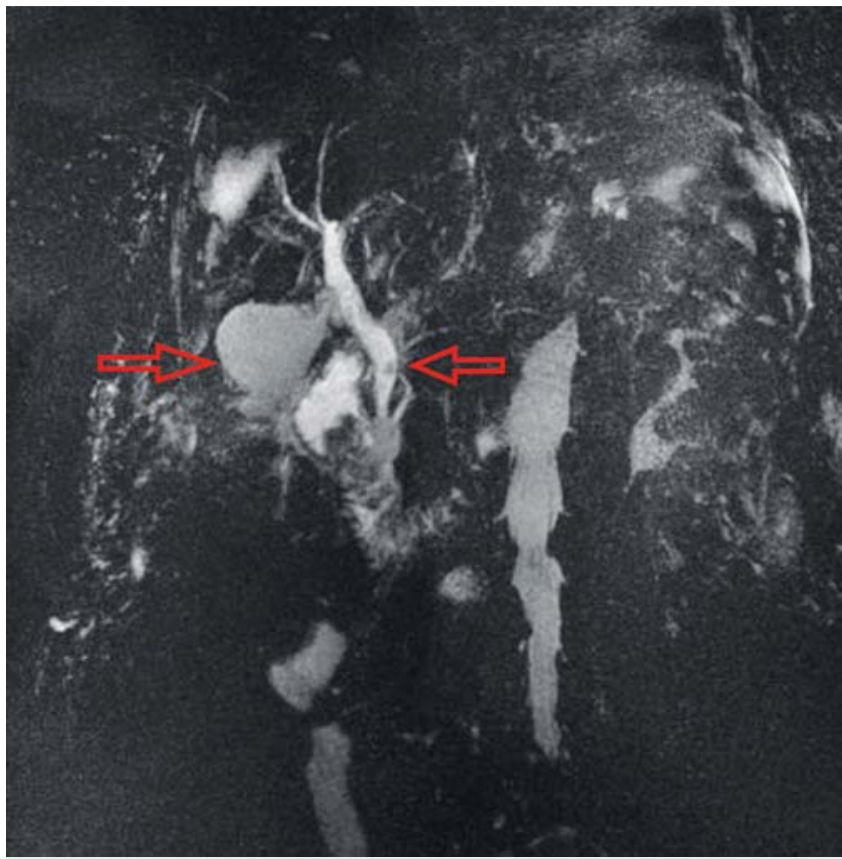

- Fig. 3 Magnetic resonance cholangiopancreatography (MRCP) demonstrating the biloma and a small stone in the common bile $\operatorname{duct}(\mathrm{CBD})$.

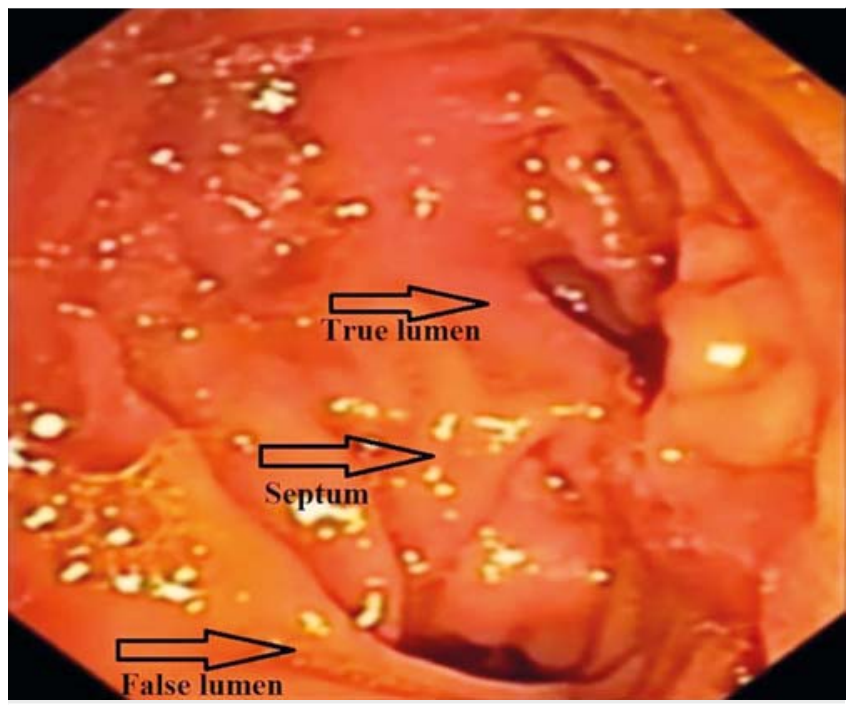

Fig. 4 Double lumen in the second portion of the duodenum.

for the duodenal lumen. An inverted diverticulum may appear like a tumor or large polyp. Differential diagnosis includes a distal choledochocele, periampullary cystic mass, or duodenal duplication cyst. Duplication cysts have no communication with the lumen and are situated in the periduodenal space. The intraduodenal diverticulum has a visible communication with

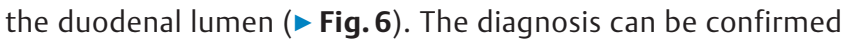
by an upper gastrointestinal series, CT with pancreatic protocol, or magnetic resonance imaging (MRI). Possible findings from CT scan are a periampullary mass, or a saccular structure with air-fluid level or oral contrast (when used), communicating 


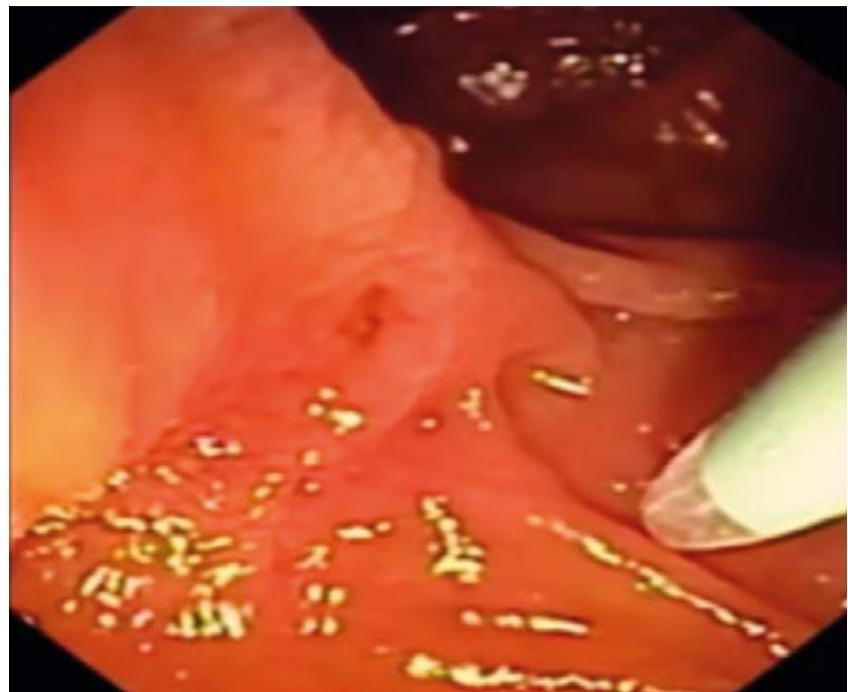

Fig. 5 Papillary orifice.

with the duodenal lumen. When oral contrast is used, the wall of the diverticulum can be seen as a low-density "flap". A collapsed or inverted diverticulum can mimic an intraluminal tumor mass or polyp, or be seen as circumscribed wall thickening. However, imaging modalities can show conflicting results as a result of the possibility of the lesion collapsing or expanding to change its appearance $[3,5]$. Surgical diverticulectomy is the traditional treatment for symptomatic patients [4]. Endoscopic snare resection or needle-knife diverticulotomy are also possible [1].

This case is unusual because of the late presentation of the congenital condition with biliary obstruction after cholecystectomy and the conflicting results of the imaging modalities. ERCP with biliary sphincterotomy was the appropriate method to solve the problem; however, ERCP can be challenging because of difficulties in finding the papillary orifice and CBD can-

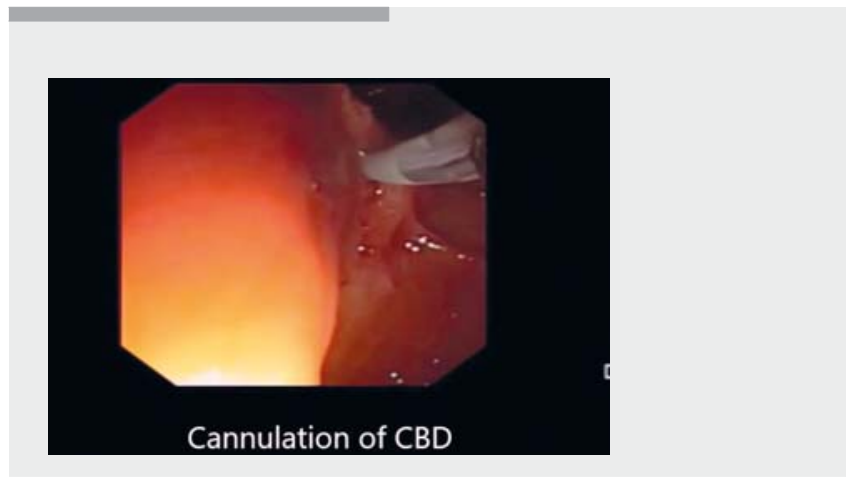

Video 1 Duodenoscopy showing double lumen in the second portion of the duodenum, cannulation of the common bile duct (CBD), biliary sphincterotomy, endoscopic papillary large balloon dilation, balloon sweeps of the CBD, and duodenal exploration using a standard gastroscope.

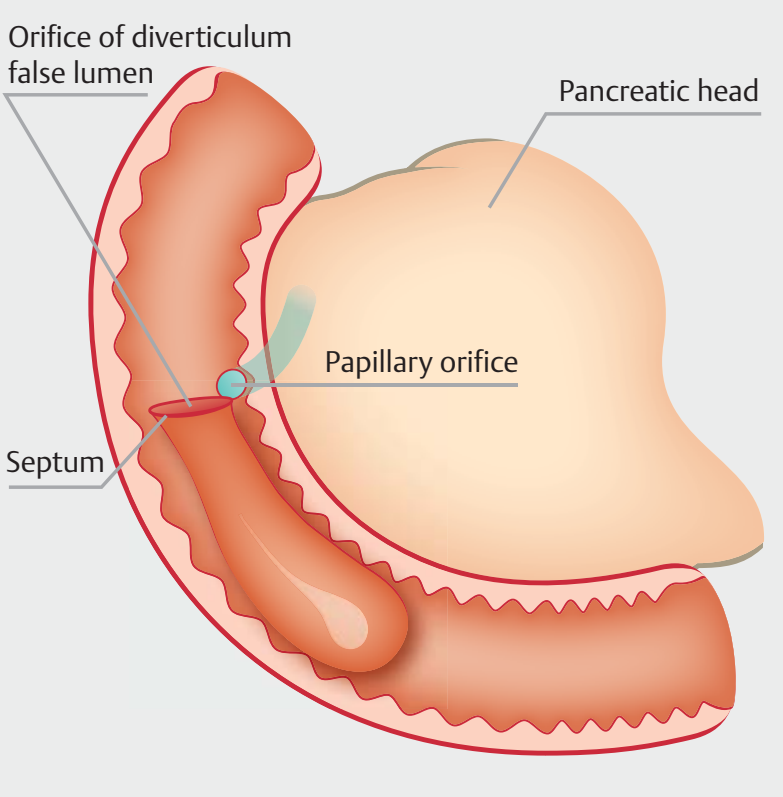

Fig. 6 Drawing demonstrating the anatomy of the intraduodenal diverticulum.

nulation as a result of the unpredictable location of the intraduodenal portion of the CBD.

\section{Competing interests}

None

\section{References}

[1] Law R, Topazian T, Baron T. Endoscopic treatment of intraluminal duodenal ("windsock") diverticulum: varying techniques from five cases. Endoscopy 2012; 44: 1161 - 1164

[2] Anand V, Provost J, Bakr M et al. Two cases of intraluminal "windsock" diverticula resulting in partial duodenal obstruction. ACG Case Rep J 2016; 3: e135

[3] Eusebio M, Ramos A, Guerreiro H. Intraluminal duodenal ("windsock") diverticulum: A rare cause of gastrointestinal bleeding. GE Port J Gastroenterol 2016; 23: 113-115

[4] Economides NG, McBurney RP, Hamilton FH. Intraluminal duodenal diverticulum in the adult. Ann Surg 1977; 185: 147-152

[5] Juanpere S, Valls L, Serra I et al. Imaging of non-neoplastic duodenal diseases. A pictorial review with emphasis on MDCT. Insights Imaging 2018; 9: $121-135$ 